\title{
AN INVERSE PROCEDURE FOR CHARACTERIZATION OF MATERIAL PARAMETERS OF PASSIVE SKELETAL MUSCLE USING FEM AND EXPERIMENTAL DATA
}

\author{
Shuailong Liu, Jianbing Sang, Yi Zhang, Luming Zhao \\ School of Mechanical Engineering, Hebei University of Technology, Tianjin, China \\ e-mail: sangjianbing@126.com
}

Guirong LiU

School of Mechanical Engineering, Hebei University of Technology, Tianjin, China, and

Department of Aerospace Engineering and Engineering Mechanics, University of Cincinnati, Cincinnati, USA

e-mail: liugr@uc.edu

\begin{abstract}
This work develops an inverse procedure which combines an improved niche genetic algorithm, finite element models and experimental data to identify material parameters of biological tissues behaving like hyperelastic materials. A novel objective function is proposed with two coefficients, which controls the contributions in a well-balanced fashion, respectively, for the small deformation stage and the large deformation stage. This allows us to obtain a global minimizer (of material constants) for the error between FEM solutions and experimental data. Moreover, simple uniaxial compression tests at two different angles $\left(0^{\circ}\right.$ and $90^{\circ}$ ) with respect to the muscle fiber orientation are performed on fresh muscle tissues. This enables us to obtain anisotropic properties of the muscle tissue from the present inverse procedure. The result shows that the proposed inverse procedure is stable and reliable to determine material constants in hyperelastic models for soft biological tissues like skeletal muscles considering anisotropy.
\end{abstract}

Keywords: niche genetic algorithm, finite element method, objective function, skeletal muscles, parameter identification

\section{Introduction}

Mechanical behavior of muscle tissues is an important field of study with different applications ranging from medicine to crashworthy vehicle design for occupants. Muscles as one of the significant sections supporting and driving animal movements, are very vulnerable to damage, especially for sportsmen and women. A comprehensive understanding of mechanical properties of muscles, including anisotropic properties, is critical to provide protective measures for athletics from muscles damage and for speedy damaged muscle recovery. Parameter identification techniques are utilized by many researchers to obtain the material constants. The majority of recent works on parameter identification are performed by comparing characteristic, experimental and calculated responses with optimization algorithms to update the material parameters systematically.

Chawla et al. (2009) combined an inverse finite element model with canonical genetic algorithms, experiments to identify material parameters of the passive muscles and obtained linear viscoelastic material constants for human muscles. The results show that the linear viscoelastic constitutive model does not properly describe characterization of the muscle tissue. Böl et al. (2014) conducted a series of experiments on muscle tissues using three different loading modes. The results provide an experimental evidence that there is an anisotropic contribution of the fiber direction to the compressive stress. Transversely isotropic, hyperelastic material models 
were usually utilized to describe the characteristic of muscles (Holzapfel et al., 2000; Gasser et al., 2006). In order to get the material parameters of the muscle tissues, Böl et al. (2012) firstly carried out a series of compressive tests with three different loading modes according to the fiber orientation of passive skeletal muscle tissue, secondly, they combined the Nelder-Mead simplex method with an inverse finite element model to fit the experimental response. However, as the complexity of the problem increases, these types of traditional optimization methods have increasingly shown limitations.

Silva et al. (2018) obtained in vivo active and passive material properties of the pubovisceralis muscle in women without pathology, with urinary incontinence and pelvic organ prolapse. They resorted an inverse finite element analysis combined with an optimization algorithm, Powell's method and finite element method (FEM). A constitutive model had been adopted, which was a modified form of the incompressible transversely isotropic hyperelastic model proposed by Martins et al. (1998).

In order to identify in vivo material parameters of the pelvic floor muscles, Yao et al. (2015) develops a non-invasive inverse approach which combines the conventional least-squares estimation method and inverse finite element model with experiments. However, the canonical least-squares needs partial derivatives in the solving process. For some practical problems, it may be very difficult to solve partial derivatives or there could be no partial derivatives at all. Since biomechanical properties of the pelvic floor muscles are essential to help us better understand the disorders in the pelvic cavity associated with those muscles, Silva et al. (2019) implemented an optimization scheme, involving a genetic algorithm (GA) and an inverse finite element analysis (FEA) to estimate the material properties of the pubovisceralis muscle. In addition, Silva conducted a comparison between the inverse procedure with the genetic algorithm to be the searching algorithm and one with Powell's algorithm to be the searching algorithm. The result shows that GA requires fewer numerical simulations to minimize the objective function in order to obtain the material parameters, when compared to Powell's algorithm. Nevertheless, Powell's algorithm presents lower function values, which means the material parameters calculated from Powell's algorithm are closer to real constants.

For a successful inverse analysis, a proper objective (or error) function needs to be defined. The definition of the objective function is crucial and can be a challenging task. The objective function adopted by Chawla for the comparison of experimental and simulated data showed a good matching at the large deformation stage, however, mismatches were found at the small deformation stage. The objective function adopted by Markus fits well at the small deformation stage, but not at the large deformation stage. This work combines those two objective functions in a single form equipped with two independent coefficients, so that the objective function can be a good measure across the entire deformation stage by adjusting those two coefficients in a proper manner.

Optimization methods also plays an important role in inverse analyses, and various types of inverse techniques have been proposed, including the use of neural networks (Deng et al., 2009), variations of genetic algorithms (Liu et al., 2001, 2002b; Ishak et al., 2001) and combinations of different methods (Liu et al., 2002a). The canonical genetic algorithm may have excessive random roaming in the searching process, and simple genetic algorithms can have a bad global convergence (Thierens, 1999; Shabbir and Omenzetter, 2016). Freutel et al. (2015) combined particle swarm optimization (PSO) with finite element analyses and applied it to identification of meniscus parameters, resulting in a low error level of $1.2 \%$. However, the canonical particle swarm optimization algorithm allows the particles learn from only the best individual, and hence some useful information of other individuals may be neglected (Du et al., 2017; Mendes et al., 2004). Zhu et al. (2010) combined the Shepard-k-Nearest method with a genetic algorithm and applied it to identify ultra-soft biological tissue parameters. Good agreements were found not only at the low and high strain rates, but also at the medium strain rate. 
To increase the chance of finding the global optimal solution, this work develops an inverse mapping program which combines the niche genetic algorithm and the finite element method (FEM) to obtain the material parameters of muscle tissues. The basic idea is to find candidates of material parameters which can be systematically updated in the optimization procedure that minimizes the objective function. The objective function measures the differences of calculated responses from finite element models and the measured responses from our own experiment data. Our proposed inverse procedure is found stable and reliable to determine the material constants for skeletal muscles considering anisotropy, based on both nonlinear FEM models and experiments.

\section{Improved niche genetic algorithm}

Real-world optimization problems are often carried out with objective functions that have multiple optima. To improve capability on the multimodal function optimization of the widely used genetic algorithm (GA), a niche technique is introduced into GA to improve the global optimization ability ( $\mathrm{Li}$ et al., 2013). This work uses an improved niche genetic algorithm (INGA) as the searching algorithm during inverse analysis. The improvement is on the selection operation in the traditional niche genetic algorithm leading to a higher global search efficiency (Im et al., 2004). The INGA has a faster convergence speed than GA and the population diversity well maintained during the evolution process. The INGA introduces the concept of niche in biology, the individual with a relatively lower fitness within the same niche is added with a decay factor on the fitness value. For others, without any operations. After that, a proportional selection operation is carried out, which has a significant impact on maintaining population diversity. To search for all the extremum points of the objective function and speed up the algorithm, INGA adopts a variable mutation probability and the variable crossover probability instead of canonical fixed values. In the INGA, the probabilities for both crossover and mutation are calculated using

$$
P_{\text {cro }}(x)=\frac{1}{1+\exp \left(x-\frac{G E N}{2}\right)} P_{\text {cro }} \quad P_{\text {mut }}(x)=\frac{1}{1+\exp \left(\frac{G E N}{2}-x\right)} P_{\text {mut }}
$$

When $P_{\text {cro }}(x)$ and $P_{m u t}(x)$ are the crossover probability and mutation probability, respectively, at the $x$-th generation, $P_{\text {cro }}$ and $P_{m u t}$ are these corresponding values at the first generation; GEN is the number of generation; $x$ is the current generation. When $x$ increases by 1 , it means that the evolution goes into the next generation. And when $x$ increases to GEN, the procedure will end. In the whole evolution, $x$ is the controller for the start and end of the procedure.

The INGA in this work is combined with Elitism preservation strategy which speeds up the convergence of the searching algorithm. In addition, a memory search scheme is introduced into the INGA to shorten the running time. The INGA proposed in this paper has a very promising performance in the field of optimization. With all these improvements, the INGA has a good performance to meet the needs in our inverse analysis.

\section{Finite element model}

Figure 1 shows a typical finite element model created for simulating our experimental set-up for inverse analysis. The skeletal muscle tissue is placed in between two platens that are connected to the 'universal testing machine' for compression tests. The type of element is CPS4R and the dimension is 3D solid element. The number of elements is 2064 and the number of nodes is 2156 . 
The top and the bottom platens are defined as a rigid body in the FEM model because these platens are made of steel that is far stiffer and harder compared to the fresh muscle tissues.

Since the microstructure of skeletal muscle tissue consists of densely packed muscle fibers which are arranged in fascicles and also have a quite nice locally arrangement in predominate alignment. The muscle could be assumed transversely isotropic with respect to the direction of the alignment, hence, in order to characterize the anisotropic behavior of the muscle tissue, the muscle tissue block is modeled as a transversely isotropic hyperelastic material. Because of the high content of water in the fresh muscle tissue, it exhibits behavior of nearly incompressible materials. Thus, a transversely isotropic, hyperelastic material model with the extension to slightly dispersed fibers by Gasser et al. (2006) was used to describe the passive behavior of the muscle tissue

$$
U=C_{10}\left(\bar{I}_{1}-3\right)+\frac{1}{D}\left(\frac{\left(J^{e l}\right)^{2}-1}{2}-\ln J^{e l}\right)+\frac{k_{1}}{2 k_{2}} \sum_{\alpha=1}^{N}\left[\exp \left(k_{2}\left\langle\bar{E}_{\alpha}\right\rangle^{2}\right)-1\right]
$$

with

$$
\bar{E}_{\alpha} \stackrel{\text { def }}{=} \kappa\left(\bar{I}_{1}-3\right)+(1-3 \kappa)\left(\bar{I}_{4(\alpha \alpha)}-1\right)
$$

where $U$ is the strain energy per unit volume; $C_{10}, D, k_{1}, k_{2}, \kappa$ are constitutive parameters; $N$ is the number of families of fibers $(N \leqslant 3)$; $\mathbf{C}$ is the right Cauchy-Green tensor; $\bar{I}_{1}$ is the first invariant of $\overline{\mathbf{C}} ; J^{e l}$ is the elastic volume ratio. The strain-like quantity $\bar{E}_{\alpha}$ characterizes deformation of the family of fibers with the mean $A_{\alpha}$; and $\bar{I}_{4(\alpha \alpha)}$ are pseudo-invariants of $\overline{\mathbf{C}}$ and $A_{\alpha}$.

The model assumes that the directions of the fibers within each family are dispersed (with rotation symmetry) about the mean preferred direction. The parameter $\kappa(0 \leqslant \kappa \leqslant 1 / 3)$ describes the level of dispersion in the fiber directions. When $\kappa=0$, the fibers are perfectly aligned (no dispersion). When $\kappa=1 / 3$, the fibers are randomly distributed and the material becomes isotropic. In this work, $D \equiv 0$. The values of the other parameters are treated as unknowns and should be obtained from our inverse procedure. These parameters are termed as to-be-determined parameters, or TBD-parameters. They are: $C_{10}, k_{1}, k_{2}$, and $\kappa$.

\section{Optimization procedure}

In our inverse procedure, the FEM model is used for forward FE analyses, by assuming these TBD-parameters. The computed responses of the muscle block are then compared with the responses measured from the experiments. We can then adjust the TBD-parameters in the FEM model, and try to find one set of the TBD-parameters that gives the FEM response closed possible to the measured response. This is the principal idea of the inverse procedure. To do this systematically, however, we need to invoke an optimization procedure that minimize the differences between FEM responses and the experimentally measured ones. For this purpose, we define the following objective function

$$
o(p)=\sum_{j=1}^{m} \sum_{i=1}^{n} \sqrt{\frac{\left({ }^{j} F_{i}^{\mathrm{exp}}-{ }^{j} F_{i}^{\text {sim }}\right)^{2}}{{ }^{j} F_{i}^{\exp }}}
$$

where $F_{i}^{\exp }$ is the experimentally measured force from a load-cell, and $F_{i}^{s i m}$ is the simulated force using our FEM model with the current set of trial TBD-parameters $P_{1}, n$ is the number of data points measured, and we set $n=180$ in this study. We set also $m=2$ that is the different fiber orientation, i.e. $0^{\circ} / 90^{\circ}$. 
(a)

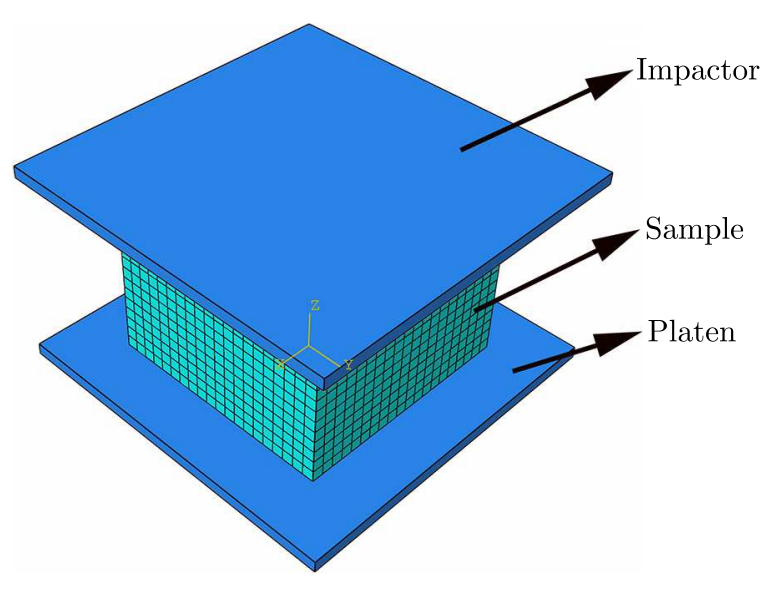

(b)
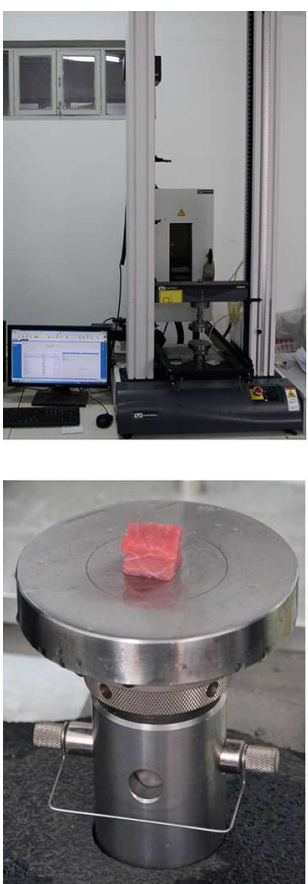

Fig. 1. (a) A typical finite element model of the block of soft muscle tissue placed in between the top and bottom steel platens. (b) Setup of the compression tests for the muscle tissue blocks

Another form of the objective function which has been adopted by other researchers is described as below

$$
o(p)=\sum_{j=1}^{m} \sum_{i=1}^{n}\left({ }^{j} F_{i}^{\exp }-{ }^{j} F_{i}^{s i m}\right)^{2}
$$

where $F_{i}^{\exp }$ is the experimentally measured force from the load-cell, and $F_{i}^{\text {sim }}$ is the simulated force using our FEM model with the current set of trial TBD-parameters $P_{2}, n$ is the number of data points measured, and we set $n=180$ in this study. We set also $m=2$ that is the different fiber orientation, i.e. $0^{\circ} / 90^{\circ}$. This paper would utilize these two forms of the objective function to identify the material parameters for muscle respectively.

\section{Inverse analysis procedure}

To perform an inverse procedure in an automated manner, a Python algorithm is coded, and it is detailed in this Section. The IDE is used to code the inverse procedure, and Python software is used as well. For FEA, the ABAQUS software is applied. It provides a solver for Python script to execute the inverse procedure. This inverse procedure could be divided into three parts, including optimization algorithm, finite element analysis and adjustment of the material parameters. The specified steps in our Python algorithm are described in Fig. 2.

\section{Experimental study}

\subsection{Experiment set-up and measurement data}

A 24-month-old adult cow weighing nearly $400 \mathrm{~kg}$ is selected as the test subject, and the hind leg is obtained from the slaughterhouse immediately after death of the animal. After that, the leg 


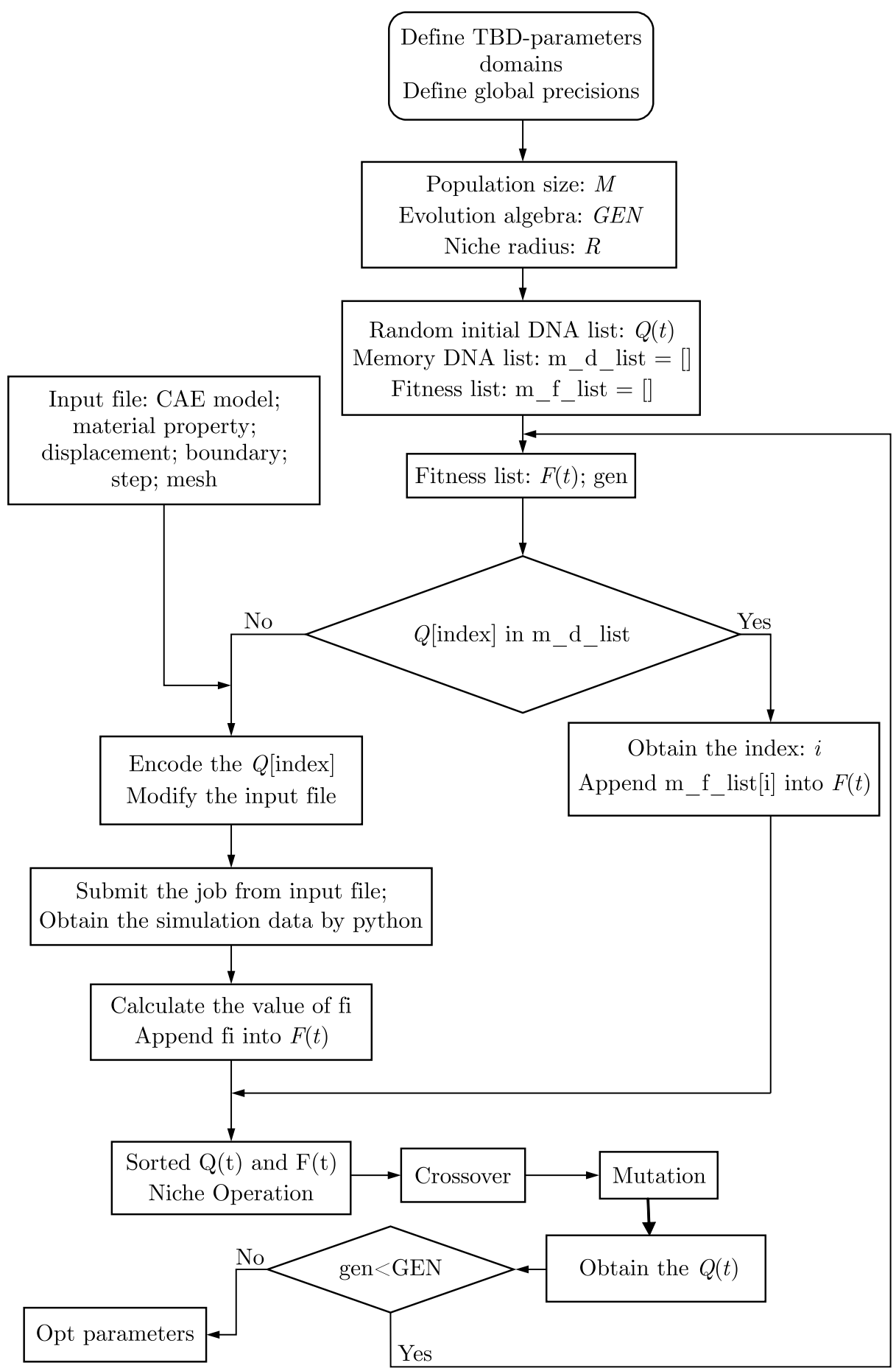

Fig. 2. Flow chart of our Python algorithm for parameter identification

has been wrapped in a physiological saline-soaked cloth and transported to the laboratory with a refrigerator at $2^{\circ} \mathrm{C}$. The saline is uniformly sprayed on the cloth every 5 minutes. However, due to the high water content of fresh muscle tissue, it is difficult to obtain the ideal cuboid geometry. Nine fresh cuboid muscle samples are cut from the approximately square bottom of the leg and stored at $2^{\circ} \mathrm{C}$ with uniform saline sprayed every 2 minutes. These tissue specimens are with two different fiber orientations, including samples whose fiber orientation are parallel to the bottom surface and samples whose fiber orientation are perpendicular to the bottom surface. The data for sample geometries are measured and summarized in Table 1. 
Table 1. Geometrical sample information for the present inverse procedure

\begin{tabular}{|c|c|c|c|c|c|c|}
\hline \multirow{2}{*}{$\begin{array}{l}\text { Tissue } \\
\text { No. }\end{array}$} & \multicolumn{3}{|c|}{$0^{\circ}$} & \multicolumn{3}{|c|}{$90^{\circ}$} \\
\hline & $\begin{array}{l}\text { Dimensions } \\
{[\mathrm{mm} \times \mathrm{mm}]}\end{array}$ & $\begin{array}{c}\text { Area } \\
A_{\phi}\left[\mathrm{mm}^{2}\right]\end{array}$ & $\begin{array}{l}\text { Height } \\
h[\mathrm{~mm}]\end{array}$ & $\begin{array}{l}\text { Dimensions } \\
{[\mathrm{mm} \times \mathrm{mm}]}\end{array}$ & $\begin{array}{c}\text { Area } \\
A_{\phi}\left[\mathrm{mm}^{2}\right]\end{array}$ & $\begin{array}{l}\text { Height } \\
h[\mathrm{~mm}]\end{array}$ \\
\hline I & $18 \times 18$ & 324 & 16.8 & $18.12 \times 20.7$ & 375.084 & 11 \\
\hline II & $19.88 \times 19.88$ & 395.2144 & 16.28 & - & - & - \\
\hline III & $21.7 \times 21.08$ & 457.436 & 17.08 & $21.3 \times 19.42$ & 413.646 & 12.8 \\
\hline IV & $16.98 \times 17.80$ & 302.244 & 15.3 & $20.8 \times 18.42$ & 383.136 & 14.98 \\
\hline V & $21.3 \times 23$ & 489.9 & 15 & $21.38 \times 21.9$ & 468.222 & 16.38 \\
\hline
\end{tabular}

All those samples are subjected to unconstrained compression tests on a universal testing machine (Instron). While the displacement $\mu$ of the top platen is predefined, the resulted force $F$ is measured and converted to the mean engineering stress $P=F / A_{\phi}$. The axial compression stretch is calculated using $\lambda=1+\mu / h$. Before testing, the samples were put in solution with 27 centigrade. All samples were tested within 7 hours after death of the animal. The specified area of tissue that was removed of the cattle is depicted in Fig. 3.

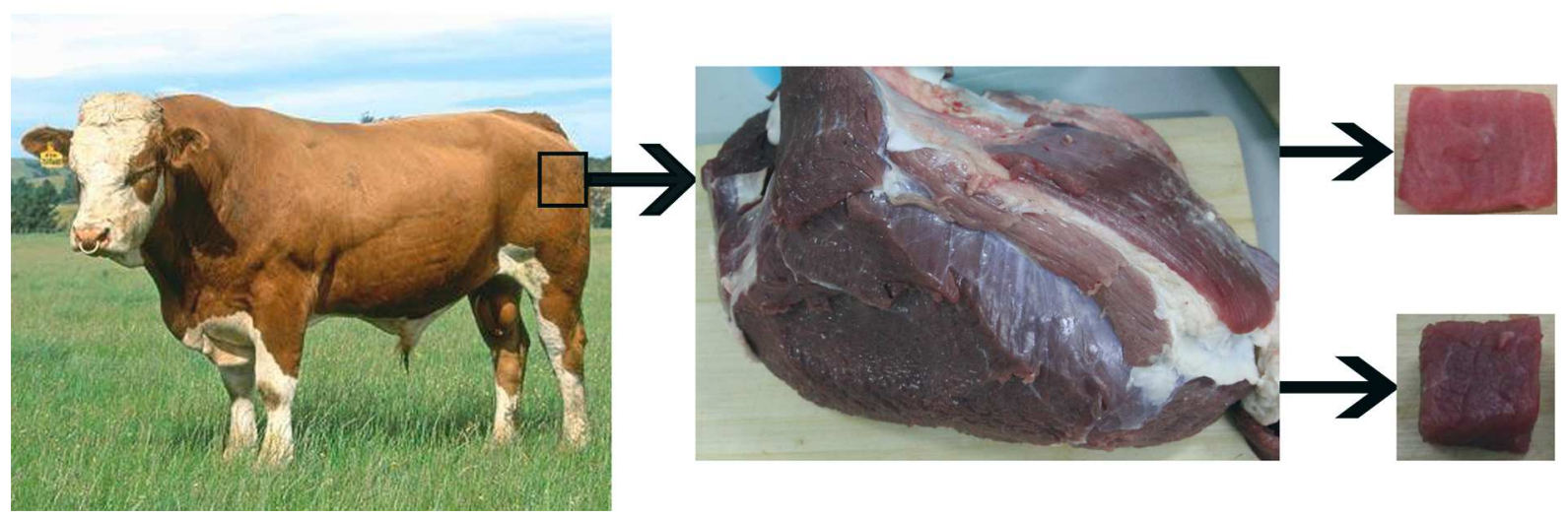

Fig. 3. The specific area on cattle where tissues were removed from

The velocity of the top platen is controlled at $0.01 \mathrm{~mm} / \mathrm{s}$. Correspondingly, the same constant velocity boundary condition is adopted in our FEM simulation. A thin layer of vegetable oil is applied between the sample and these two platens in order to reduce friction. The data of $\lambda$ obtained from the experiments are in a range of 0.65 to 1 and are recorded. The experimental set-up is shown in Fig. 1b.

Figure 4a plots these curves obtained from our experiments, for specimen with fiber orientation parallel to the loading direction. The red dotted curve is the curve for the averaged data of 5 samples. Figure $4 \mathrm{~b}$ plots these curves obtained from our experiments, for the specimen with fibers perpendicular to the loading direction. The red dotted curve is the curve for the averaged data of 5 samples. From Figs. $4 \mathrm{a}$ and $4 \mathrm{~b}$, it could be found that $P$ and $\lambda$ have a non-linear relationship, and $P$ increases with a increase of $\lambda$. It also could be found that the muscle tissue has a greater ability to resistance the compressive deformation in the direction which is perpendicular to the fiber orientation. In view of the high level of compression applied in this study, the average relative standard deviations are small and take values of $0.33670,0.33031$ for fiber directions $0^{\circ}$ and $90^{\circ}$, respectively.

The experimental data are fed into the inverse procedure discussed in Section 5. Additional data needed for our inverse procedure are given in Table 2. In order to model the behavior on the interface between the fresh tissue and those two steel platens, the friction there is considered. However, we do not really know the friction coefficient. We thus decide to treat the friction 

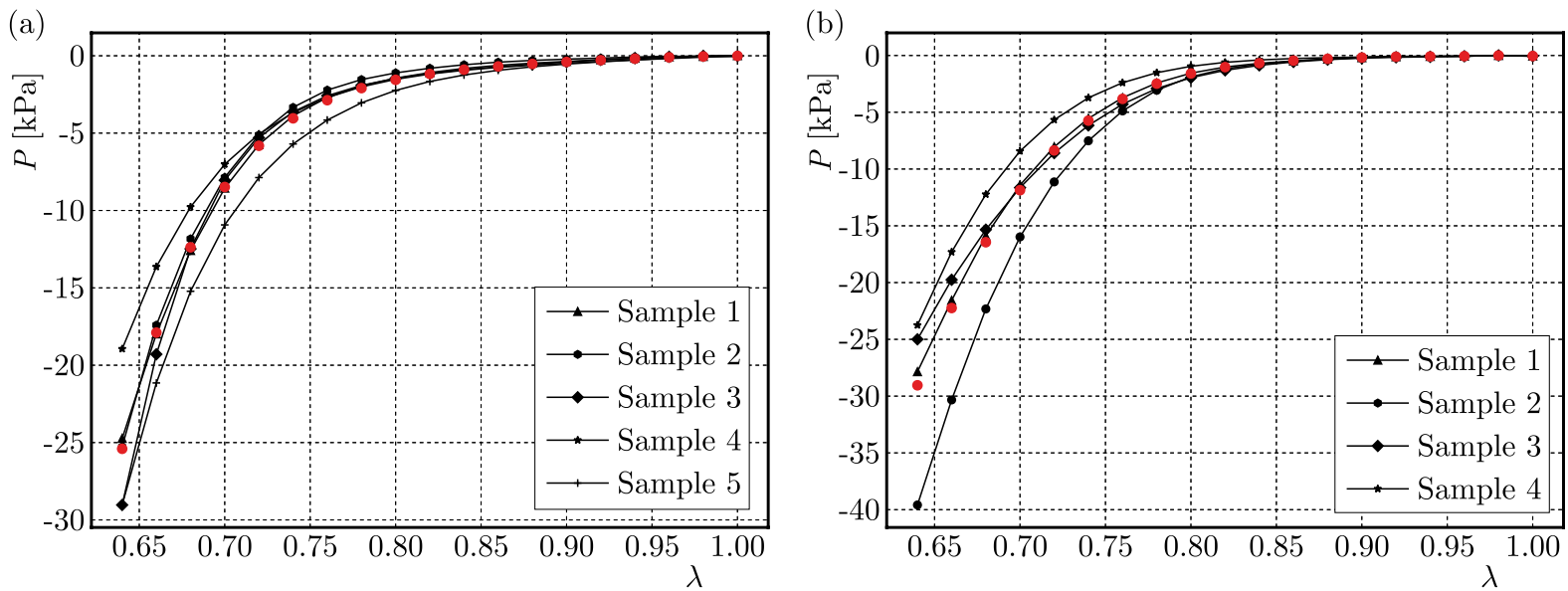

Fig. 4. Compressive stress-stretch responses of 10 samples. The loading direction is parallel (a) and perpendicular (b)to the tissues fiber direction. The red dotted curve is for the average data

Table 2. Improved niche genetic algorithm input parameters, including parameter domains for the progress searching and other parameters to be defined before the procedure

\begin{tabular}{|l|c|}
\hline \multicolumn{1}{|c|}{ Description of function } & Value \\
\hline \hline Number of evolutionary algebra & 30 \\
\hline Population size & 100 \\
\hline Number of real coded variables & 5 \\
\hline Variables iterated (TBD-parameters) & $C_{10}, k_{1}, k_{2}, \kappa, \alpha$ \\
\hline Lower and upper bound of $C_{10}$ & $C_{10}^{\text {min }}=10 \mathrm{~Pa}, C_{10}^{\text {max }}=2 \cdot 10^{3} \mathrm{~Pa}$ \\
\hline Lower and upper bound of $k_{1}$ & $k_{1}^{\text {min }}=1 \cdot 10^{3} \mathrm{~Pa}, k_{1}^{\text {max }}=700 \cdot 10^{3} \mathrm{~Pa}$ \\
\hline Lower and upper bound of $k_{2}$ & $k_{2}^{\text {min }}=10, k_{2}^{\text {max }}=180$ \\
\hline Lower and upper bound of $\kappa$ & $\kappa^{\text {min }}=0.001, \kappa^{\text {max }}=0.28$ \\
\hline Lower and upper bound of friction & $\alpha^{\text {min }}=0.001, \alpha^{\text {max }}=0.020$ \\
\hline Cross over probability & $P_{\text {cro }}=0.9$ \\
\hline Mutation probability & $P_{\text {mut }}=0.12$ \\
\hline Niche radius & 10 \\
\hline
\end{tabular}

coefficient $\alpha$ as one of the TBD-parameters in our inverse procedure, therefore, we know have five TBD-parameters in total, they are $C_{10}, k_{1}, k_{2}, \kappa, \alpha$. Those five TBD-parameter and their domains as well as some other arguments needed in the inverse procedure are shown in Table 2.

\subsection{Inverse determined parameters and their assessment}

Using our automated inverse procedure defined in Section 5 and the experimental data obtained in Section 6.1, the TBD-parameters are then determined, which are termed as $D$-parameters for convenience. When using objective function (4.1), the $D$-parameters are in set $P_{1}$, and those $D$-parameters obtained using objective function (4.2) are in the set $P_{2}$.

To quantitatively assess the accuracy of these $D$-parameters, we next use them in our ABAQUS model to compute the stress-stretch responses. When the $D$-parameters in $P_{1}$ are used, the computed stress-stretch responses together with the average experimentally obtained curve are plotted in Fig. 5a for the case of fiber orientation parallel to the loading direction. The same is plotted in Fig. 5a, but for the case of fiber orientation perpendicular to the loading direction.

When the $D$-parameters in $P_{2}$ are used, the computed stress-stretch responses are plotted in Fig. 5 a, together with the experimentally obtained curve, for the case of fiber orientation parallel 

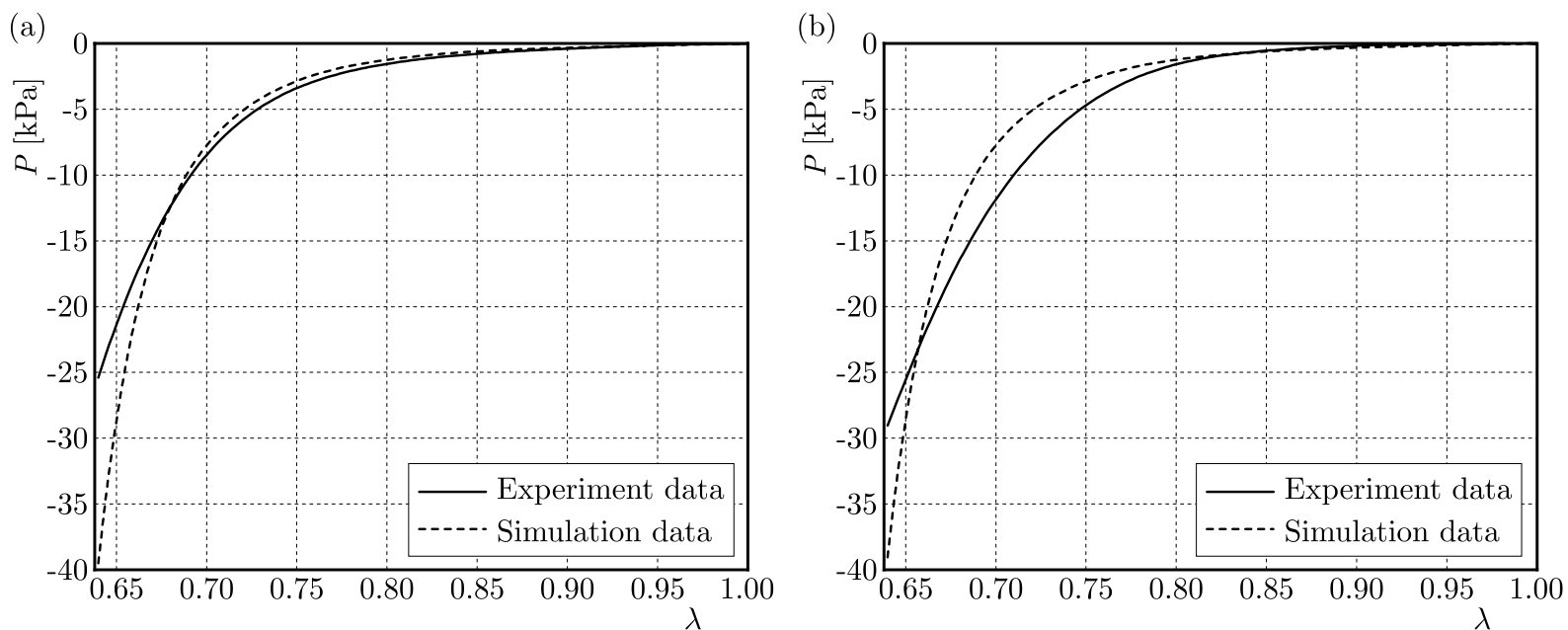

Fig. 5. FEM results obtained using the $D$-parameters when equation (4.1) is used as the objective function in our inverse procedure, in comparison with the experiment data. The loading direction is parallel (a) and perpendicular (b) to the fiber direction

to the loading direction. The same is plotted in Fig. 5b, but for the case of fiber orientation perpendicular to the loading direction.

From our experimental observations (see, Figs. 5a and 5b), it could be found that the computed response and the experimental response matches well at the small deformation stage, but not at the large deformation stage. The deviation between the computed responses and the measured responses is getting bigger and bigger with an increase of the deformation.

From our experimental observations (see, Figs. 6a and 6b), it could be found that the computed response and the experimental response matches well at the large deformation stage, but not at the small deformation stage. From the comparison of Fig. 4b and Fig. 5b, it could be found that the advantage of those two forms of objective functions appear at different stages respectively. The matching result in the entire domain for the calculated and measured response, not the matching result in a certain partial domain, is a measure of quality of the objective function.
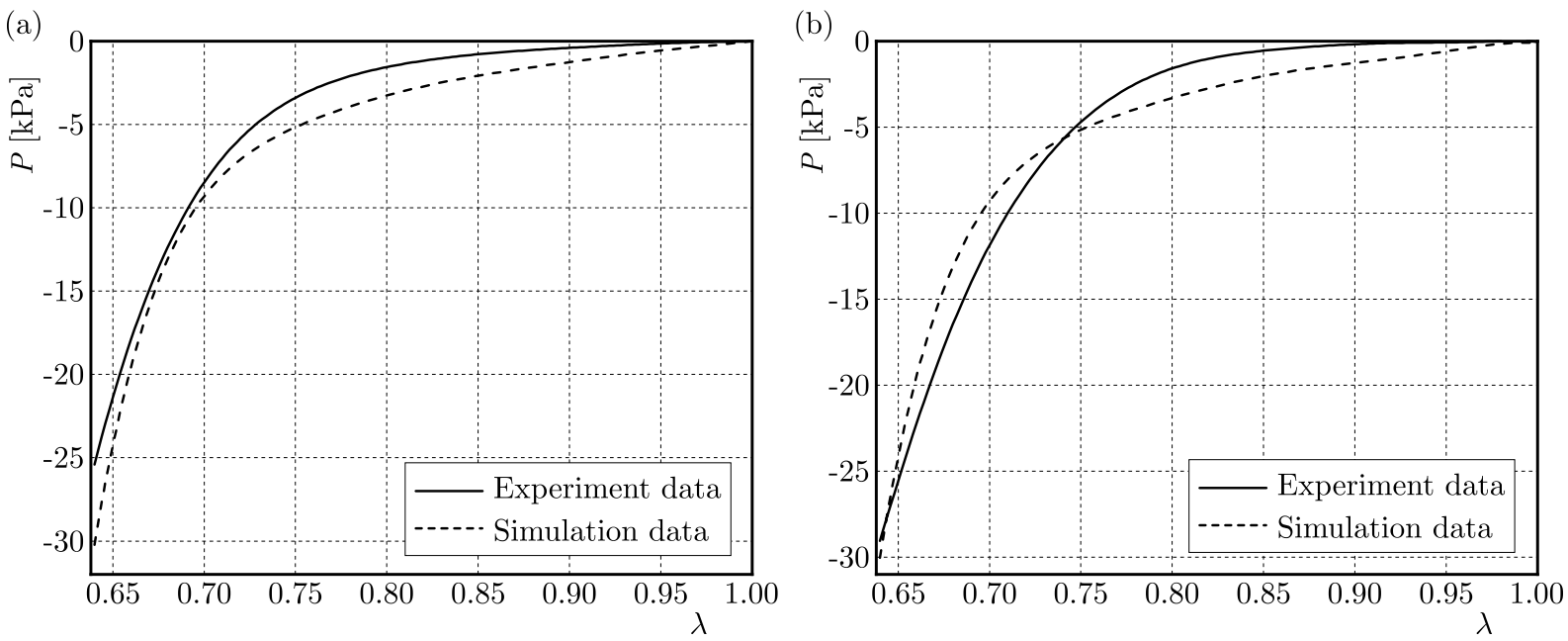

Fig. 6. FEM results obtained using the $D$-parameters when equation (4.2) is used as the objective function in our inverse procedure, in comparison with the experiment data. The loading direction is parallel (a) and perpendicular (b) to the fiber direction 
Obviously, those two objective functions discussed above cannot be called a preferred objective function. In order to enhance the performance of the objective function in the whole domain, we combine those two objective functions above. Two controllable coefficients are applied to strengthen the versatility of the objective function. Its form can be expressed as follows

$$
o(p)=k_{1} \sum_{j=1}^{m} \sum_{i=1}^{n} \sqrt{\frac{\left({ }^{j} F_{i}^{\text {exp }}-{ }^{j} F_{i}^{\text {sim }}\right)^{2}}{{ }^{j} F_{i}^{e x p}}}+k_{2} \sum_{j=1}^{m} \sum_{i=1}^{n}\left({ }^{j} F_{i}^{e x p}-{ }^{j} F_{i}^{s i m}\right)^{2}
$$

Our objective function has now two terms controlled by two coefficients. The first part controls the mismatch between the computed response and the measured response during the small deformation stage, while the second term controls that at the large deformation stage.

When $k_{1}=1$ and $k_{2}=0$, objective function (4.3) is transformed to be function (4.1); when $k_{1}=0$ and $k_{2}=1$, objective function (4.3) is transformed to be function (4.2). For different problems, the best matching result in the whole domain for the calculated and experimental response can be obtained by adjusting the coefficients of those two terms.

However, how to determine the coefficient of those two items has become an urgent problem to be solved. In order to solve it, firstly, we set those two coefficients to be $k_{1}=1$ and $k_{2}=0$, the calculated response and the measured response are fed into function (4.2) to calculate $A$, and the two coefficients are set $k_{1}=0$ and $k_{2}=1$, secondly, the calculated and measured response are fed into function (4.2) to calculate $B$, thirdly, the define a function to represent the relation between $k_{1}$ and $k_{2}$

$$
\frac{A}{B}=\frac{k_{2}}{k_{1}}
$$

In this paper, the ratio of $A$ and $B$ is rounded and defined by $d \approx A / B$. When $d>1, k_{1}=1$, $k_{2}=d$; when $d<1, k_{1}=1 / d, k_{2}=1$. In this paper, $d \approx 5$, which means $k_{1}=1, k_{2}=5$. Those two values of the coefficients are fed into objective function (4.3), and function (4.3) is updated into the inverse process, the $D$-parameters are in the set $P_{3}$. When the $D$-parameters in $P_{3}$ are used, the computed stress-stretch response is plotted in Fig. 7a together with the experimentally obtained curve for the case of fiber orientation parallel to the loading direction. The same is plotted in Fig. 7b, but for the case of fiber orientation perpendicular to the loading direction.
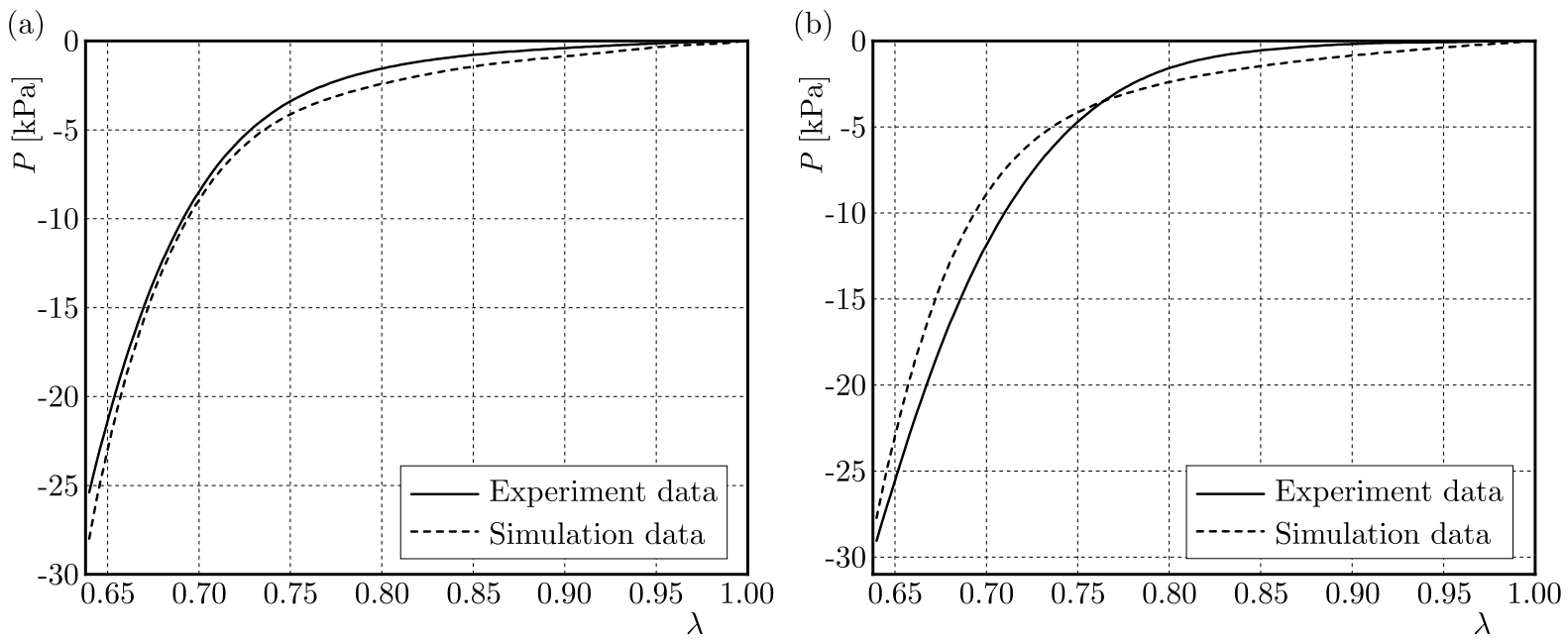

Fig. 7. FEM results obtained using the $D$-parameters when equation (4.3) is used as the objective function in our inverse procedure, in comparison with the experiment data. The loading direction is parallel (a) and perpendicular (b) to the fiber direction

From the comparison of Fig. 5a and Fig. 6a, it could be found that the degree of the agreement between the calculated response and the average experimental response is higher in Fig. 5b. The 
results obtained from the comparison of Fig. 5b and Fig. 6b shows that Fig. 6b has a better agreement. To quantify the degree of matching between the simulated response and the average test response, a comparison study for those two set of $D$-parameters is conducted. In doing so, a general error function is defined for quantitative examination

$$
\text { error }=\frac{1}{m n} \sqrt{\sum_{j=1}^{m} \sum_{i=1}^{n}\left(\frac{{ }^{j} F_{i}^{\text {exp }}-{ }^{j} F_{i}^{\text {sim }}}{{ }^{j} F_{i}^{\text {exp }}}\right)^{2}}
$$

The calculated value of the error represents the difference between the $D$-parameters obtained from the inverse procedure and the real constants. The lower the error value, the better the calculated responses matching the measured responses and the closer the $D$-parameters are to the real constants. The $D$-parameters obtained from the inverse procedure represent the anisotropy of the muscle, and the errors calculated from the error function are shown in Table 3. According to Table 3, we can see that the error for the set $P_{2}$ is lower than the error for the set $P_{1}$, which means that the set $P_{2}$ is closer to the real constants. Correspondingly, objective function (4.3) is more suitable for the inverse procedure in this work.

Table 3. The $D$-parameters of muscle tissues and the error value

\begin{tabular}{|c|c|c|c|c|c|c|c|}
\hline Parameters & $C_{10}$ & $D$ & $k_{1}$ & $k_{2}$ & $\kappa$ & $\alpha$ & error (6.1) \\
\hline \hline$P_{1}$ & 0.000304 & 0 & 0.057693 & 48 & 0.207049 & 0.012402 & 0.244670 \\
\hline$P_{2}$ & 0.001333 & 0 & 0.494938 & 19 & 0.140389 & 0.140383 & 0.216041 \\
\hline$P_{3}$ & 0.0008902 & 0 & 0.222694 & 23 & 0.160694 & 0.014370 & 0.059913 \\
\hline
\end{tabular}

The two sets of $D$-parameters $P_{1}$ and $P_{2}$ are obtained from the inverse procedure by utilizing objective functions (4.1) and (4.2), respectively, and $P_{3}$ is obtained from the inverse procedure by using objective function (4.3) with $k_{1}=1$ and $k_{2}=5$. Table 3 shows clearly that the set $P_{3}$ has a much lower error value, meaning that it is closer to the real material parameters. The results shown in Figs. 7a and 7b also support this finding. It can be concluded that the objective function proposed in this paper has a good performance when applied in the parameter identification of passive skeletal muscles undergoing large deformation. This research further shows that the transverse isotropic hyperelastic material model (HGO) has a very good performance in describing the anisotropic mechanical properties of the muscle tissue.

\section{Conclusion}

An inverse procedure has been developed to identify material parameters of passive skeletal muscles. The basic idea of this reverse process is to combine an improved niche genetic algorithm with the inverse finite element method by adjusting the material parameters to achieve the best fit between the calculated response and the experimental measurement. In the reverse procedure, the purpose of the finite element method is to establish a simulation model and obtain simulation data. Moreover, the improved niche genetic algorithm is employed by the reverse procedure to search the optimal material parameters within the designed space. It can be seen from the results that the mean engineering stress and the axial compression stretch have a strong non-linear relationship regardless of whether the fiber orientation is perpendicular or parallel to the loading direction. When the angle between the fiber orientation and the loading direction increases, the ability to resist deformation is enhanced, which is consistent with the results of Böl et al. (2012).

Concerning the experimental compressive response of passive muscle tissue, the results are partially in line with those obtained by Loocke et al. (2006) for the orientation dependent compression of fresh porcine muscle tissue. The final conclusion can be achieved as follows: 
- The approach of combining the improved niche genetic algorithm, FEM model, and the experimental data is an effective approach to identify nonlinear and anisotropic material properties of passive skeletal muscles under compression conditions.

- A modified objective function has been proposed in the work. The result of the inverse procedure utilizing the modified objective function gives a much smaller error compared with the existing objective function. This type of a multiple piece objective function proposed in this work can be extended for different types of identification problems by properly adjusting these coefficients for different loading stages.

- The inverse procedure proposed in this work may be applicable to tissues subjected to other types of loading conditions, and also to other soft tissues that obey the hyperelastic constitutive model.

\section{Acknowledgement}

This paper is supported by Key Project of Natural Science Foundation of China (Grant No. 11832011) Tianjin Excellent Special Correspondent Project (Grant No. 16JCTPJC53100) and Scientific Research Key Project of Hebei Province Education Department (Grand No. ZD2016083).

\section{References}

1. Böl M., Ehret A.E., Leichsenring K., Weichert C., Kruse R., 2014, On the anisotropy of skeletal muscle tissue under compression, Acta Biomaterialia, 10, 7, 3225-3234, DOI: 10.1016/j.actbio.2014.03.003

2. Böl M., Kruse R., Ehret A.E., Leichsenring K., Siebert T., 2012, Compressive properties of passive skeletal muscle - The impact of precise sample geometry on parameter identification in inverse finite element analysis, Journal of Biomechanics, 45, 15, 2673-2679

3. Chawla A., Mukherjee S., Karthikeyan B., 2009, Characterization of human passive muscles for impact loads using genetic algorithm and inverse finite element methods, Biomechanics and Modeling in Mechanobiology, 8, 1, 67-76

4. Deng B., Tan K.B.C., Lu Y., Zaw K., Zhang J., Liu G.R., Geng J.P., 2009, Inverse identification of elastic modulus of dental implant-bone interfacial tissue using neural network and FEA model, Inverse Problems in Science and Engineering, 17, 8, 1073-1083, DOI: $10.1080 / 17415970903063151$

5. Du W.B., Ying W., Yan G., Zhu Y.B., CaO X.B., 2017, Heterogeneous Strategy Particle Swarm Optimization, IEEE Transactions on Circuits and Systems II Express Briefs, 64, 4, 467471, DOI: $10.1109 /$ TCSII.2016.2595597

6. Freutel M., Galbusera F., Ignatius A., Dürselen L., 2015, Material properties of individual menisci and their attachments obtained through inverse FE-analysis, Journal of Biomechanics, 48, 8, 1343-1349, DOI: 10.1016/j.jbiomech.2015.03.014

7. Gasser T.C., Ogden R.W., Holzapfel G.A., 2006, Hyperelastic modelling of arterial layers with distributed collagen fibre orientations, Journal of the Royal Society Interface, 3, 6, 15-35

8. Holzapfel G.A., Gasser T.C., Ogden R.W., 2000, A new constitutive framework for arterial wall mechanics and a comparative study of material models, Journal of Elasticity and the Physical Science of Solids, 61, 1-3, 1-48

9. Im C.H., Kim H.K., Jung H.K., ChOi K., 2004, A novel algorithm for multimodal function optimization based on evolution strategy, IEEE Transactions on Magnetics, 40, 2, 1224-1227, DOI: 10.1109/TMAG.2004.824805

10. Ishak S.I., Liu G.R., Lim S.P., Shang H.M., 2001, Experimental study on employing flexural wave measurement to characterize delamination in beams, Experimental Mechanics, 41, 2, 157-164 
11. Li K.S., Yu X.L., Zhang W.S., Dong W.Y., 2013, Multimodal function optimization algorithm based on improved niche evolutionary algorithm, Journal of System Simulation, 13, 25, 6, 1170-1175

12. LiU G.R., HAN X., LAM K.Y., 2002a, A combined genetic algorithm and nonlinear least squares method for material characterization using elastic waves, Computer Methods in Applied Mechanics and Engineering, 191, 17, 1909-1921

13. LiU G.R., HAN X., XU Y.G., LAM K.Y., 2001, Material characterization of functionally graded material by means of elastic waves and a progressive-learning neural network, Composites Science and Technology, 61, 10, 1401-1411, DOI: 10.1016/S0266-3538(01)00033-1

14. LiU G.R., LAM K.Y., HAN X., 2002b, Determination of elastic constants of anisotropic laminated plates using elastic waves and a progressive neural network, Journal of Sound and Vibration, 252, 2, 239-259

15. Loocke M.V., Lyons C.G., Simms C.K., 2006, A validated model of passive muscle in compression, Journal of Biomechanics, 39, 16, 2999-3009

16. Martins J.A.C., Pires E.B., Salvado R., Dinis P.B., 1998, A numerical model of passive and active behavior of skeletal muscles, Computer Methods in Applied Mechanics and Engineering, 151, 3-4, 419-433, DOI: 10.1016/S0045-7825(97)00162-X

17. Mendes R., Kennedy J., Neves J., 2004, The fully informed particle swarm: simpler, maybe better, IEEE Transactions on Evolutionary Computation, 8, 3, 204-210

18. Shabbir F., Omenzetter P., 2016, Model updating using genetic algorithms with sequential niche technique, Engineering Structures, 120, 166-182

19. Silva E., Parente M., Brandăo S., Mascarenhas T., Natal J.R., 2019, Characterizing the biomechanical properties of the pubovisceralis muscle using a genetic algorithm and the finite element method, Journal of Biomechanical Engineering, 141, 1

20. Silva M.E.T., Parente M.P.L., Brandăo S., Mascarenhas T., 2018, Characterization of the passive and active material parameters of the pubovisceralis muscle using an inverse numerical method, Journal of Biomechanics, 71, DOI: 10.1016/j.jbiomech.2018.01.033

21. Thierens D., 1999, Scalability Problems of Simple Genetic Algorithms, MIT Press

22. Yao R., Du L., Zhu L., Xiang Z., 2015, An inverse approach to identifying the in vivo material parameters of female pelvic floor muscles using MRI data, Science China Life Sciences, 58, 3, 305-308, DOI: 10.1007/s11427-014-4771-6

23. Zhu F., Jin X., Guan F., Zhang L., 2010, Identifying the properties of ultra-soft materials using a new methodology of combined specimen-specific finite element model and optimization techniques, Materials and Design, 31, 10, 4704-4712, DOI: 10.1016/j.matdes.2010.05.023 